\title{
Some Entanglement Features Exhibited by Two, Three and Four Qubits Interacting with an Environment in a Non-Markovian Regime
}

\author{
A. Hamadou-Ibrahim ${ }^{1}$, A.R. Plastino ${ }^{2,3 *}$, and C. Zander ${ }^{1}$ \\ ${ }^{1}$ Physics Department, University of Pretoria, Pretoria 0002, South Africa \\ ${ }^{2}$ Instituto de Física Teórica y Computacional Carlos I, \\ Departamento de Física, Universidad de Granada, Granada, Spain, EU \\ ${ }^{3}$ National University La Plata, UNLP-CREG-CONICET, \\ Casilla de Correo 727, 1900 La Plata, Argentina.
}

(Dated: November 21, 2009)

\begin{abstract}
We explore various aspects of the quantum entanglement dynamics of systems of two, three and four qubits interacting with an environment at zero temperature in a non-Markovian regime, as described by the paradigmatic model recently studied by Bellomo, Lo Franco, and Compagno [Bellomo et al. Phys. Rev. Lett. 99 (2007) 160502]. We consider important families of initial states for the alluded systems. The average, typical entanglement evolution associated with each of these families is determined, and its relation with the evolution of the global degree of mixedness of the multi-qubit system is explored. For three and four qubits we consider the family of initial states equivalent under local unitary transformations to the $|G H Z\rangle$ and $|W\rangle$ states, and compare their average behavior with the average behavior exhibited by initial maximally entangled twoqubits states. Furthermore, in the case of two qubits, the evolution of other manifestations of entanglement, related to measurable quantities, is also investigated. In particular, we consider the Mintert-Buchleitner concurrence lower bound and an entanglement indicator based upon the violation of local uncertainty relations.
\end{abstract}

PACS numbers: 03.67.Mn, 03.65.Yz

Keywords: Entanglement Dynamics, Decoherence

\footnotetext{
* Corresponding author: arplastino@maple.up.ac.za
} 


\section{INTRODUCTION}

Entanglement and decoherence constitute two fundamental ingredients in the present understanding of the quantum fabric of the physical world [1-4]. The diverse manifestations of quantum entanglement are nowadays the focus of intense theoretical and experimental research efforts. Quantum entanglement plays an essential role, for example, in connection with the emergence of the classical picture of the macroscopic world from a quantum mechanical substratum [4]. It also provides a deep physical justification for the basic tenets of quantum statistical mechanics [5]. Moreover, intriguing recent developments suggest that quantum entanglement may be relevant for explaining the origin of the macroscopic "arrow of time" [6]. On the other hand, the actual creation and manipulation of multipartite entangled states in the laboratory lie at the heart of spectacular new technological developments, such as quantum computation [2,3], and quantum metrology [7]. Quantum entanglement and decoherence are closely related to each other. In fact, the phenomenon of decoherence basically consists of a family of effects that occur due to the interaction (and associated entanglement-development) between quantum systems and their environments $[3,4]$. Physical systems in Nature aren't usually in complete isolation and interact with their environments in some way. As a consequence of this interaction, in most cases, some entanglement develops between the system and the environment (there are special instances, however, where a system may interact with another system without developing entanglement with it). The system-environment entanglement leads to the suppression of typical quantum features of the system, such as the interference between different system's states. This constitutes the basic idea behind the "decoherence program" for explaining the quantum-to-classical transition [4].

The amount of entanglement between the different constituent parts of a multipartite quantum system tends to decrease as the alluded composite system undergoes decoherence. This decay of entanglement has recently attracted the interest of many researchers [8-17] because it constitutes one of the most difficult obstacles that have to be overcome to develop quantum technologies requiring the controlled manipulation of entangled states [3]. A remarkable recent discovery is that, in some cases, entanglement can disappear completely in finite times. This effect is known as entanglement sudden death (ESD) [8-15] and has been observed experimentally by Almeida et al. [16]. Besides its theoretical importance, ESD 
is also a phenomenon of considerable relevance from the practical point of view, because the actual implementation of quantum computation and other quantum information tasks crucially depends on the longevity of entanglement in multiqubits systems.

To study the consequences of the interaction between a quantum mechanical system and its surroundings the system must be treated as an open quantum system (see [18] for an excellent, comprehensive and updated discussion on open quantum systems). In order to succeed in the development of useful devices for quantum information processing it is imperative to achieve a systematic characterization and understanding of the abovementioned effects arising from the interaction with the environment. The aim of the present work is to explore some typical features of the entanglement dynamics of systems of independent qubits each interacting with a reservoir in a regime where the non-Markovian effects are important. In other words, we are going to consider reservoirs whose correlation times are greater than, or of the same order as, the relaxation time over which the state of the system changes [18]. Interesting previous work on the entanglement dynamics of two-qubits systems interacting with an environment in the non-Markovian regime has been reported by Bellomo, Lo Franco, and Compagno (BFC) in a recent series of papers [10-12] (see also [14]). A remarkable phenomenon studied by BFC is that, for certain initial states, there is entanglement sudden death and afterwards entanglement sudden revival. BFC focused their attention on initial states described by density matrices of the " $X$-form", which admit a particularly elegant analytical treatment. We will extend the work by BFC in various directions. We will investigate the average, typical entanglement dynamics associated with some relevant families of initial states of two-qubits, three-qubits, and four-qubits systems. We will explore the relation between the time evolution of the amount of entanglement of these multi-qubit systems and their degrees of mixedness. In the case of two qubits, we will also investigate the possibility of detecting the disappearance of entanglement and its subsequent revival using two recently advanced entanglement indicators. 


\section{QUBITS-RESERVOIR MODEL}

We are going to consider the paradigmatic model discussed in [10], which is based on the "qubit + reservoir" Hamiltonian,

$$
H=\omega_{0} \sigma_{+} \sigma_{-}+\sum_{k} \omega_{k} b_{k}^{\dagger} b_{k}+\left(\sigma_{+} B+\sigma_{-} B^{\dagger}\right)
$$

where $B=\sum_{k} g_{k} b_{k}, \omega_{0}$ denotes the transition frequency of the two-level system (that is, the qubit) and $\sigma_{\mp}$ stands for the system's raising and lowering operators. The reservoir is represented as a set of field modes, $b_{k}^{\dagger}$ and $b_{k}$ being the concomitant creation and annihilation operators associated with the $k$-mode. These field modes are characterized by frequencies $\omega_{k}$ and coupling constants $g_{k}$ with the two-level system. The Hamiltonian (1) may describe, for instance, a qubit consisting of the excited and ground electronic states of a two-level atom that interacts with the quantized electromagnetic modes of a high-Q cavity. The assumed effective spectral density of the reservoir is

$$
J(\omega)=\frac{1}{2 \pi} \frac{\gamma_{0} \lambda^{2}}{\left(\omega-\omega_{0}\right)^{2}+\lambda^{2}},
$$

where $\gamma_{0}$ and $\lambda$ are positive parameters with dimensions of inverse time. The parameter $\lambda$, giving the width of $J(\omega)$, is related to the reservoir's correlation time $\tau_{B}$ by $\tau_{B} \approx \lambda^{-1}$. The parameter $\gamma_{0}$ is connected with the system's relaxation time $\tau_{R}$ via $\tau_{R} \approx \gamma_{0}^{-1}$ (see [10] for details). In the strong coupling, non-Markovian regime we have $\gamma_{0}>\lambda / 2$. The Hamiltonian (1) was previously studied by Garraway [19] who obtained the analytical solution for the concomitant dynamics. The dynamics of the single qubit is described by the density matrix

$$
\rho(t)=\left(\begin{array}{cc}
\rho_{11}(0) P_{t} & \rho_{10}(0) \sqrt{P_{t}} \\
\rho_{01}(0) \sqrt{P_{t}} & \rho_{00}(0)+\rho_{11}(0)\left(1-P_{t}\right)
\end{array}\right),
$$

where $\rho_{i j}(0)$ are the initial density matrix elements of the qubit and the function $P_{t}$ is given (in the non-Markovian regime [10]) by

$$
P_{t}=e^{-\lambda t}\left[\cos \left(\frac{d t}{2}\right)+\frac{\lambda}{d} \sin \left(\frac{d t}{2}\right)\right]^{2}
$$

with $d=\sqrt{2 \gamma_{0} \lambda-\lambda^{2}}$. The time evolution of two non-interacting qubits, each of them in contact with an independent reservoir and, consequently, individually evolving according to 
(3), is then given by a time dependent statistical operator whose elements with respect to the computational basis $\{|1\rangle \equiv|11\rangle,|2\rangle \equiv|10\rangle,|3\rangle \equiv|01\rangle,|4\rangle \equiv|00\rangle\}$ are [10]

$$
\begin{aligned}
& \rho_{11}^{T}(t)=\rho_{11}^{T}(0) P_{t}^{2} ; \quad \rho_{22}^{T}(t)=\rho_{22}^{T}(0) P_{t}+\rho_{11}^{T}(0) P_{t}\left(1-P_{t}\right), \\
& \rho_{33}^{T}(t)=\rho_{33}^{T}(0) P_{t}+\rho_{11}^{T}(0) P_{t}\left(1-P_{t}\right) ; \quad \rho_{44}^{T}(t)=1-\left[\rho_{11}^{T}+\rho_{22}^{T}+\rho_{33}^{T}\right], \\
& \rho_{12}^{T}(t)=\rho_{12}^{T}(0) P_{t}^{3 / 2} ; \quad \rho_{13}^{T}(t)=\rho_{13}^{T}(0) P_{t}^{3 / 2}, \\
& \rho_{14}^{T}(t)=\rho_{14}^{T}(0) P_{t} ; \quad \rho_{23}^{T}(t)=\rho_{23}^{T}(0) P_{t}, \\
& \rho_{24}^{T}(t)=\sqrt{P_{t}}\left[\rho_{24}^{T}(0)+\rho_{13}^{T}(0) P_{t}\left(1-P_{t}\right)\right], \\
& \rho_{34}^{T}(t)=\sqrt{P_{t}}\left[\rho_{34}^{T}(0)+\rho_{12}^{T}(0) P_{t}\left(1-P_{t}\right)\right],
\end{aligned}
$$

with $\rho_{i j}^{T}(t)=\rho_{j i}^{T *}(t)$ (that is, the matrix $\rho^{T}(t)$ is Hermitian). It is possible to derive equations similar to (5) corresponding to the time dependent density matrix associated with the evolution of a set of $N$ non-interacting qubits each of them interacting with its "own" reservoir. In Section V we are going to consider the three-qubit case.

\section{TYPICAL ENTANGLEMENT DYNAMICS FOR TWO QUBITS}

\section{A. Generation of Random States Within a Family of Initial States.}

In order to investigate the average features characterizing the entanglement dynamics associated with a given family of initial states we compute the average properties of the concomitant evolutions. To determine these averages we generate random initial states (within the alluded family) uniformly distributed according to the Haar measure [20, 21]. We shall consider a family of maximally entangled initial states, a family of partially entangled pure initial states all sharing the same amount of entanglement, and a family of Werner states.

To study the typical, average behavior of the entanglement dynamics of a pair of qubits evolving from an initial maximally entangled state we represent the initial states $\left|\Psi_{e}\right\rangle$ as $[20]$

$$
\left|\Psi_{e}\right\rangle=\left(I_{2} \otimes U_{1}\right)\left|\Psi_{0}\right\rangle
$$

where $\left|\Psi_{0}\right\rangle=\frac{1}{\sqrt{2}}(|01\rangle+|10\rangle), I_{2}$ denotes the two-dimensional identity matrix and $U_{1}$ is a 
unitary matrix on $S U(2)$. This unitary matrix can be conveniently parameterized as

$$
U_{1}=\left(\begin{array}{cc}
-\sin \vartheta e^{-i \theta_{2}} & \cos \vartheta e^{i \theta_{1}} \\
\cos \vartheta e^{-i \theta_{1}} & \sin \vartheta e^{i \theta_{2}}
\end{array}\right)
$$

where $\theta_{1,2} \in[0,2 \pi]$ and $\vartheta=\in[0, \pi / 2]$. In terms of the three parameters $\theta_{1}, \theta_{2}$, and $\vartheta$, the maximally entangled state reads,

$$
\left|\Psi_{e}\right\rangle=\frac{1}{\sqrt{2}}\left[\begin{array}{c}
\cos \vartheta e^{i \theta_{1}} \\
\sin \vartheta e^{i \theta_{2}} \\
-\sin \vartheta e^{-i \theta_{2}} \\
\cos \vartheta e^{-i \theta_{1}}
\end{array}\right]
$$

where $\left|\Psi_{e}\right\rangle$ is represented as a column vector in terms of its coefficients with respect to the computational basis. To generate the initial states we generate random (single-qubit) unitary matrices $U$ uniformly distributed according to the Haar measure. The angles $\theta_{i}$ are generated randomly such that they are uniformly distributed in $[0,2 \pi]$, while $\vartheta$ is distributed in the interval $[0, \pi / 2]$ according to the distribution $\sin (2 \vartheta)$. This distribution can be obtained by setting $\vartheta=\arcsin \left[\epsilon^{1 / 2}\right]$ with $\epsilon$ uniformly distributed in $[0,1]$.

More generally, random pure states exhibiting a fixed, prescribed amount of entanglement can be generated using the representation

$$
\left|\Psi_{\alpha}\right\rangle=\left(I_{2} \otimes U_{1}\right)\left(\sqrt{1-\alpha^{2}}|01\rangle+\alpha|10\rangle\right)
$$

which leads to the parameterization

$$
\left|\Psi_{\alpha}\right\rangle=\left[\begin{array}{c}
\sqrt{1-\alpha^{2}} \cos \vartheta e^{i \theta_{1}} \\
\sqrt{1-\alpha^{2}} \sin \vartheta e^{i \theta_{2}} \\
-\alpha \sin \vartheta e^{-i \theta_{2}} \\
\alpha \cos \vartheta e^{-i \theta_{1}}
\end{array}\right]
$$

where one can change the degree of entanglement by using different values of $\alpha$. For instance the value $\alpha=\frac{1}{\sqrt{2}}$ will give the maximally entangled states above. The parameters $\theta_{1,2}$ and $\vartheta$ appearing in (10) have to be generated in the same way as in the case of the maximally entangled states. Note that we are not sampling the full space $C P^{3}$ of pure states of two-qubits. We are only sampling a family of states equivalent under local unitary transformations to a given, prescribed state. 


\section{B. Maximally Entangled Initial States.}

In this section we are going to explore the typical, average entanglement dynamics corresponding to maximally entangled initial states. To this end we generate random maximally entangled initial states according to the procedure described in the previous section and compute, for different times, the averages of the concurrence $C$ and the linear entropy $S_{L}=\frac{4}{3}\left[1-\operatorname{Tr}\left(\rho^{2}\right)\right]$. The average values of the concurrency (left) and that of the linear entropy (right) are depicted in Fig. 1 as a function of the dimensionless quantity $\gamma_{0} t$. In all our computations we set $\lambda=0.01 \gamma_{0}$. The dispersion $\Delta C=\left(\left\langle C^{2}\right\rangle-\langle C\rangle\right)^{\frac{1}{2}}$ is also plotted in Fig. 1. The dispersion $\Delta C$ is relatively small compared with $\langle C\rangle$, meaning that the behavior of the average $\langle C\rangle$ is representative of the typical entanglement dynamics corresponding to the family of initial maximally entangled states. The same occurs with the other families of initial states considered in the present work. (Note that $\Delta C$ is not the error in the curve $\langle C\rangle_{\text {vs. }} \gamma_{0} t$. The error in this and the other curves depicted in this work is not appreciable at the scale of the figures).

Even though, on average, the concurrence does vanish at certain times, it doesn't stay equal to zero during finite time intervals. In other words, the finite time intervals of vanishing entanglement before the entanglement revivals, that are observed for certain initial states, are not a feature characterizing the average entanglement dynamics. This observation is going to be of relevance when we later compare the entanglement dynamics of two qubits with the entanglement behaviors corresponding to three qubits or four qubits.

It is a well-known trend that the amount of entanglement exhibited by quantum states of a bipartite system tends to decrease as we consider states with increasing degrees of mixedness (see [21] and references therein). In point of fact, all two-qubits states with a linear entropy larger than 8/9 have zero entanglement (that is, are separable). The abovementioned general trend connecting entanglement and mixedness is consistent with the average behaviors of the concurrence and the linear entropy during the first half of the initial period of entanglement decrease observed in Fig. 1. During this first part of the two-qubits evolution the concurrence (and, consequently, the amount of entanglement) decreases while the degree of mixedness increases. However, after this first phase of the evolution the pattern changes: the concurrence and the mixedness increase or decrease together. In particular, during the entanglement revivals, the entanglement and the degree of mixedness of the 
two-qubit system tend to adopt their maximum values at the same time.
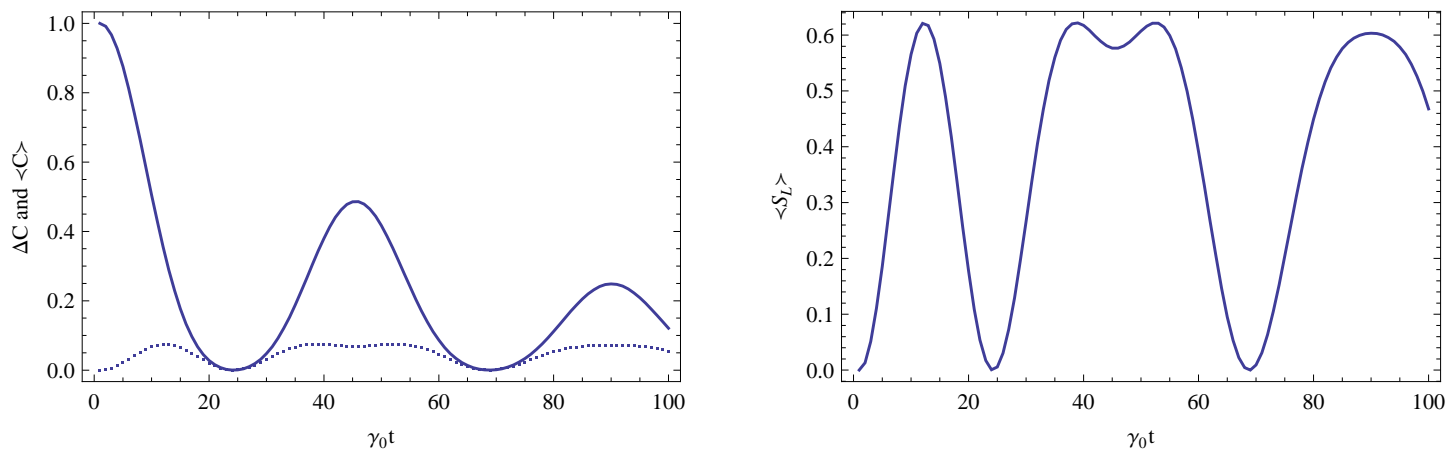

FIG. 1: The average value of the concurrence $\langle C\rangle$ and its dispersion $\left(\left\langle C^{2}\right\rangle-\langle C\rangle^{2}\right)^{1 / 2}$ (left) and the average value of the linear entropy (right), against the quantity $\gamma_{0} t$, for maximally entangled initial states. All depicted quantities are dimensionless.

When considering the relationship between the amount of entanglement and the degree of mixedness of two-qubits states, the maximally entangled mixed states (MEMS) play an important role. The MEMS [28] states are two-qubits states that have the maximum possible value of the concurrence for a given degree of mixture and their density matrix is given by

$$
\rho_{M E M S}=\left(\begin{array}{cccc}
g(\gamma) & 0 & 0 & \gamma / 2 \\
0 & 1-2 g(\gamma) & 0 & 0 \\
0 & 0 & 0 & 0 \\
\gamma / 2 & 0 & 0 & g(\gamma)
\end{array}\right)
$$

where

$$
g(\gamma)= \begin{cases}\gamma / 2, & \gamma \geq 2 / 3 \\ 1 / 3, & \gamma<2 / 3\end{cases}
$$

Some aspects of the entanglement dynamics of our two-qubits system can be illuminated if we consider now the trajectory followed by this system in the mixedness-concurrence plane, and compare this trajectory with the curve corresponding to the MEMS states. Fig. 2 shows a plot of the average value of the concurrence against the average value of the linear entropy (continuous line) for maximally entangled initial states. The curve in the $\left(S_{L}-C\right)$-plane corresponding to the concurrence $C_{m e m s}$ associated with maximally entangled mixed states (MEMS) of linear entropy $S_{L}$ is also depicted (dotted line). 


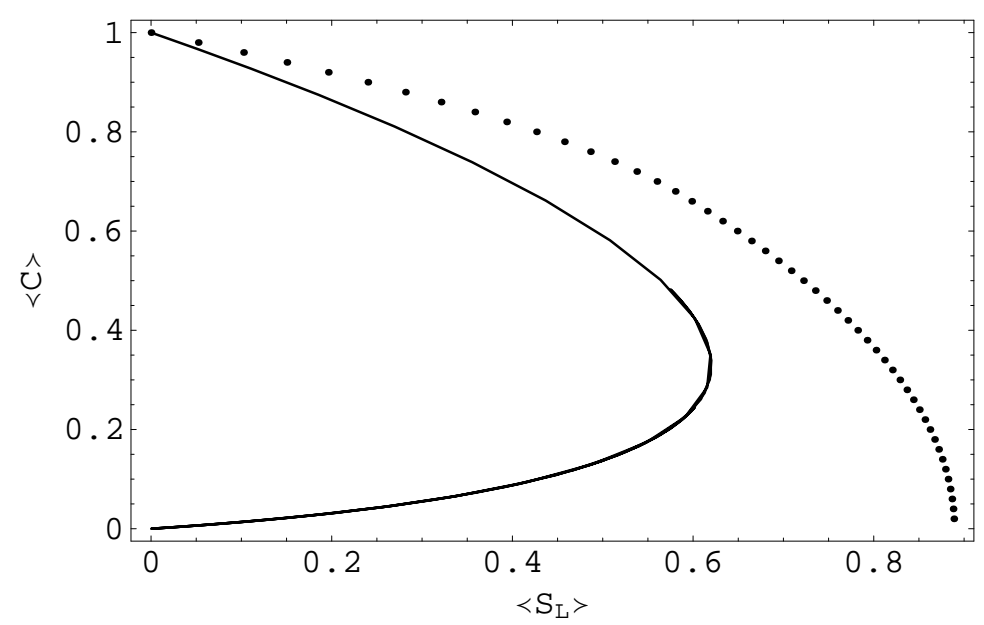

FIG. 2: The average concurrence against the average linear entropy for maximally entangled initial states (continuous line) and the concurrence of the MEMS (dotted line) against the linear entropy. All depicted quantities are dimensionless.

It can be appreciated in Fig. 2 that the average trajectory in the $\left(S_{L}-C\right)$-plane associated with maximally entangled initial states has two branches: an upper branch that stays relatively close to the MEMS curve and a lower branch that departs drastically from the MEMS. During the first phase of entanglement decrease, the average evolution associated with maximally entangled initial states describes the complete trajectory depicted in Fig. 2, starting with states of maximum entanglement and zero mixedness and ending with states of zero entanglement and zero mixedness. During the periods of entanglement revival, the average evolution follows the lower branch, first in the direction corresponding to an increase of entanglement and mixedness, and then in the opposite direction. During the first entanglement revival the two-qubit states reach the point of maximum $\left\langle S_{L}\right\rangle$ in the $\left\langle S_{L}\right\rangle-\langle C\rangle$ curve, and retrace part of the upper branch. In the second and later entanglement revivals, the two-qubits states remain in the lower branch.

The time averaged amount of entanglement exhibited by an evolving composite system is also an interesting quantity to investigate. This quantity has already been considered in previous studies, in various contexts [29, 30]. Entanglement is a valuable resource, and the time average of the entanglement of a system during a given time interval provides a rough idea of the amount of entanglement that is available at an instant of time chosen at random during the alluded interval. We have computed numerically the time average of the 
concurrence of the two-qubit system,

$$
\langle C\rangle_{t}=\frac{1}{\tau} \int_{0}^{\tau} C(t) d t
$$

where $\tau$ is the time when the concurrence vanishes for the second time (that is, $\tau$ corresponds to the end of the first revival event). In particular we computed $\langle C\rangle_{t}$ taking the Bell states

$$
\begin{aligned}
& \left|\beta_{00}\right\rangle=\frac{1}{\sqrt{2}}(|00\rangle+|11\rangle) \\
& \left|\beta_{01}\right\rangle=\frac{1}{\sqrt{2}}(|01\rangle+|10\rangle) \\
& \left|\beta_{10}\right\rangle=\frac{1}{\sqrt{2}}(|00\rangle-|11\rangle) \\
& \left|\beta_{11}\right\rangle=\frac{1}{\sqrt{2}}(|01\rangle-|10\rangle)
\end{aligned}
$$

as initial states, obtaining the values $\langle C\rangle_{t}=0.225336$ for $\left|\beta_{00}\right\rangle$ and $\left|\beta_{10}\right\rangle$ and $\langle C\rangle_{t}=0.376867$ for $\left|\beta_{01}\right\rangle$ and $\left|\beta_{11}\right\rangle$. A numerical search for the maximum value of $\langle C\rangle_{t}$ among evolutions

starting with a maximally entangled initial state yielded a maximum value $\langle C\rangle_{t}^{(\text {max. })}=$ 0.376867. This maximum value is achieved by the states $\left|\beta_{01}\right\rangle$ and $\left|\beta_{11}\right\rangle$.

\section{Partially Entangled Pure Initial States.}

The average behavior corresponding to pure, partially entangled initial states is qualitatively similar to the one corresponding to maximally entangled initial states, but with a $\left\langle S_{L}\right\rangle-\langle C\rangle$ trajectory obviously starting with states of concurrence less than one and zero mixedness (that is $S_{L}=0$ ). The average behavior, as a function of $\gamma_{0} t$, of the concurrence is depicted in Fig. 3 for initial states having the same entanglement as the state

$$
|\Phi\rangle=\alpha|00\rangle+\sqrt{1-\alpha^{2}}|11\rangle
$$

for $\alpha^{2}=1 / 3$. It can be seen in Fig. 3 that the finite time intervals of zero entanglement disappear when we consider the average behavior of the abovementioned states. This is in clear contrast with the behavior of some particular initial states belonging to the above family (see the individual case also depicted in Fig. 3) whose associated trajectories show rather long intervals with zero entanglement [10]. 


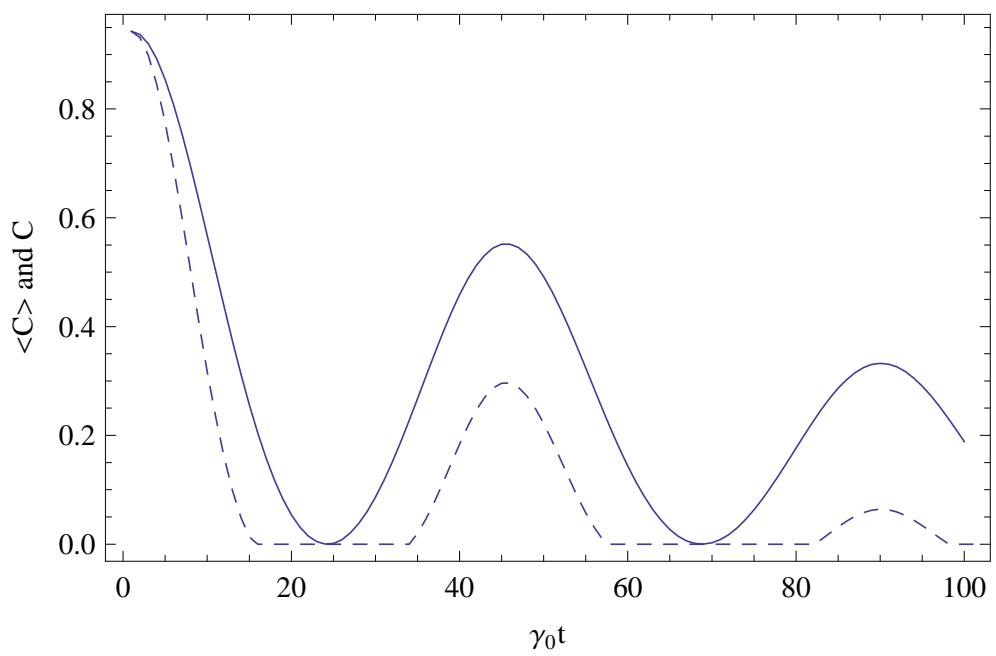

FIG. 3: The concurrence for the initial state (18) (dotted line) and the average value of the concurrence for initial partially entangled pure states having the same entanglement as (18) (continuous line) as a function of $\gamma_{0} t$. In both cases $\alpha^{2}=1 / 3$. All depicted quantities are dimensionless.

We obtained an analytical expression linking $S_{L}$ and $C$ during the evolution associated with individual partially entangled initial states of the form (18). The trajectory on the $\left(S_{L}-C\right)$-plane corresponding to these initial states is given by

$$
S_{L}=\left(\frac{C^{2}}{4 \alpha^{2}\left(1-\alpha^{2}\right)}-\frac{C}{2 \alpha \sqrt{1-\alpha^{2}}}\right) .
$$

The average trajectories on the $\left(S_{L}-C\right)$-plane of initial pure states with the same entanglement (concurrence) as the state (18) are depicted in Fig. 4 for different values of $\alpha$. 


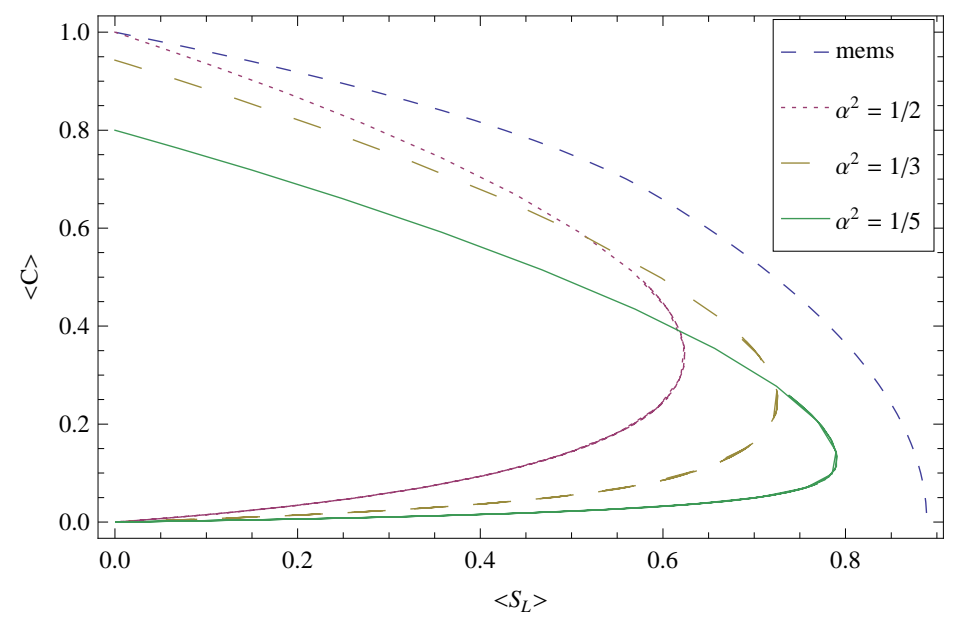

FIG. 4: Trajectories in the $\left(\left\langle S_{L}\right\rangle-\langle C\rangle\right)$-plane corresponding to families of initial partially entangled pure states with a given amount of entanglement. All depicted quantities are dimensionless.

It transpires from Fig. 4 that the smaller the initial entanglement, the larger the maximum degree of mixedness achieved by the two-qubit system during its evolution. Another trend that can be observed in Fig. 4 is that the smaller the initial entanglement, the closer the second branch of the average trajectory is to the $\langle C\rangle=0$ line. This, of course, is related to the increasing length of the time intervals of zero entanglement corresponding to initial pure states of decreasing entanglement.

\section{Initial Mixed States of Two Qubits.}

Now we are going to consider the entanglement dynamics associated with mixed initial states of the Werner form

$$
\rho=\gamma|\Psi\rangle\langle\Psi|+\frac{1-\gamma}{4} I
$$

where $0 \leq \gamma \leq 1, I$ is the $4 \times 4$ identity matrix and $|\Psi\rangle$ is a maximally entangled pure state of the form (6). The state $\rho$ represents a mixture of a maximally entangled pure state and the completely mixed state $\frac{I}{4}$. The state $\rho$ is entangled for $\gamma>1 / 3$.

In order to study the typical, average behavior of initial mixed states of the form (20) we randomly generated the maximally entangled states $|\Psi\rangle$ (according to the procedure explained in Section III.A) and then computed the average properties associated with the evolutions corresponding to the family of states (20). 


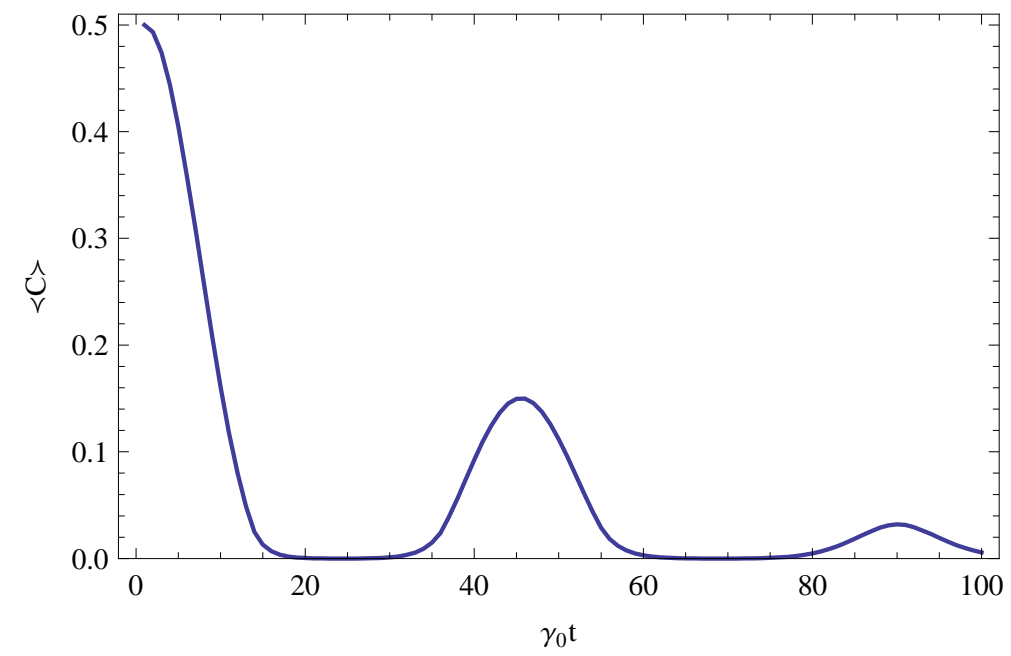

FIG. 5: Average value of the concurrence as a function of $\gamma_{0} t$ for the initial mixed states $\gamma|\Psi\rangle\langle\Psi|+$ $\frac{1-\gamma}{4} I$ with $\gamma=\frac{2}{3}$. All depicted quantities are dimensionless.

The results obtained, for $\gamma=\frac{2}{3}$, are summarized in Figures 5 and 6 . We can see in these Figures that the behavior of the initial mixed states (20) shares some general features with the behavior of the maximally entangled initial states considered previously. There is, however, one important difference (aside from the fact that the trajectory on the $\left\langle S_{L}\right\rangle-\langle C\rangle$ plane starts from an initial state of partial entanglement and finite linear entropy). The lower branch of the trajectory on the $\left\langle S_{L}\right\rangle-\langle C\rangle$ plane depicted in Fig. 6 has a long, almost horizontal part associated with states of very little, almost zero entanglement. This section of the lower branch corresponds to the time intervals between entanglement death and entanglement revivals in Fig. 5. This means that the existence of finite intervals of basically zero entanglement before entanglement revivals constitutes a typical, average property exhibited by the family of states (20). 


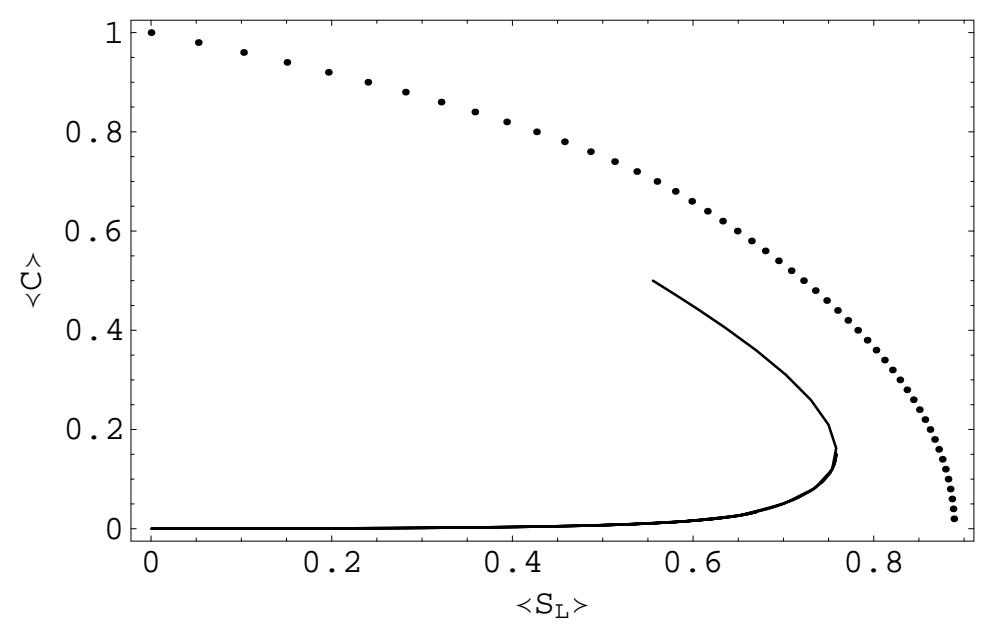

FIG. 6: Average value of the concurrence $\langle C\rangle$ against the average linear entropy $\left\langle S_{L}\right\rangle$ for the same family of initial mixed states considered in Fig. 5 (continuous line) and the concurrence of the maximally entangled mixed states $C_{m e m s}$ against $S_{L}$ (dotted line). All depicted quantities are dimensionless.

\section{BEHAVIOR OF SOME ENTANGLEMENT INDICATORS FOR TWO- QUBITS STATES}

Quantum entanglement gives rise to diverse peculiar properties of entangled states, such as the violation of Bell inequalities [2]. However, not all entangled states are endowed with all these special features. Consequently, it is of considerable relevance not only to determine the amount of entanglement associated with given quantum states, but also to explore which entangled states do exhibit (and which do not) the different entanglement-related manifestations. The recent study by Bellomo et al. [12] of the violation of Bell inequalities by two-qubits interacting with an environment constituted a notable contribution, within the context of the entanglement dynamics of open systems, to the abovementioned line of inquiry. On a similar vein, non-classical entropic inequalities satisfied by the time dependent state of two qubits evolving according to (5) was examined in [15].

Besides its theoretical interest, the exploration of which states do exhibit the different entanglement-related features is of considerable practical interest because some of the alluded entanglement manifestations can be used to construct entanglement-indicators based on measurable quantities. In this Section we are going to consider two such entanglement 
indicators: the Minternt-Buchleitner lower bound for the squared concurrence, and an entanglement indicator based on local uncertainty relations [27].

\section{A. Minternt-Buchleitner Lower bound for the squared concurrence.}

A remarkable indicator of entanglement for quantum states $\rho_{A B}$ of bi-partite systems has been recently advanced by Minternt and Buchleitner (MB) [22, 23],

$$
E_{M B}\left[\rho_{A B}\right]=2 \operatorname{Tr}\left[\rho_{A B}^{2}\right]-\operatorname{Tr}\left[\rho_{A}^{2}\right]-\operatorname{Tr}\left[\rho_{B}^{2}\right] .
$$

The MB entanglement indicator $E_{M B}$ is particularly interesting because, as was shown by $\mathrm{MB}$, it is an experimentally measurable quantity that provides a lower bound for the squared concurrence of $\rho_{A B}$,

$$
C^{2}\left[\rho_{A B}\right] \geq E_{M B}\left[\rho_{A B}\right]
$$

Last, but certainly not least, the indicator $E_{M B}$ is a practical, mathematically simple to compute quantity.

It is interesting to examine the behavior of $E_{M B}$ in a time dependent setting. The behavior of $E_{M B}$ is compared with that of the squared concurrence $C^{2}$ in Fig. 7 where both quantities are plotted against $\gamma_{0} t$ for the initial state $\frac{1}{\sqrt{2}}(|00\rangle+|11\rangle)$. The average values of the concurrence squared $C^{2}$ and of the MB lower bound were also computed for the evolutions corresponding to initial maximally entangled, randomly generated states. The results obtained are depicted in Fig. 8.

The quantum states considered in Figures 7 and 8 have $\operatorname{Tr}\left[\rho_{A}^{2}\right]=\operatorname{Tr}\left[\rho_{B}^{2}\right]$ and, consequently, for these states we can write $E_{M B}\left[\rho_{A B}\right]=2\left(\operatorname{Tr}\left[\rho_{A B}^{2}\right]-\operatorname{Tr}\left[\rho_{A}^{2}\right]\right)$. One can verify in Figures 7 and 8 that, indeed, the quantity $E_{M B}$ constitutes a lower bound for $C^{2}$. The results depicted in Fig. 7 indicate that, for the initial state $\frac{1}{\sqrt{2}}(|00\rangle+|11\rangle)$, the lower bound $E_{M B}$ provides a reasonably good estimate of the amount of entanglement exhibited by the two qubits during the first period of entanglement decrease. The quantity $E_{M B}$ is also able to detect the first entanglement revival, at least during the time interval around the peak value exhibited by $C^{2}$ in this revival (this interval corresponds, approximately, to one third of the duration of the first revival). On the contrary, $E_{M B}$ doesn't detect the second or later entanglement revivals. 
As for the typical behavior of the lower bound $E_{M B}$ corresponding to initial maximally entangled states, on average, $\left\langle E_{M B}\right\rangle$ provides a reasonable estimate for the squared concurrence during the first half of the first time interval of entanglement decrease. However, $\left\langle E_{M B}\right\rangle$ doesn't detect the subsequent entanglement revivals (see Fig. (8)).

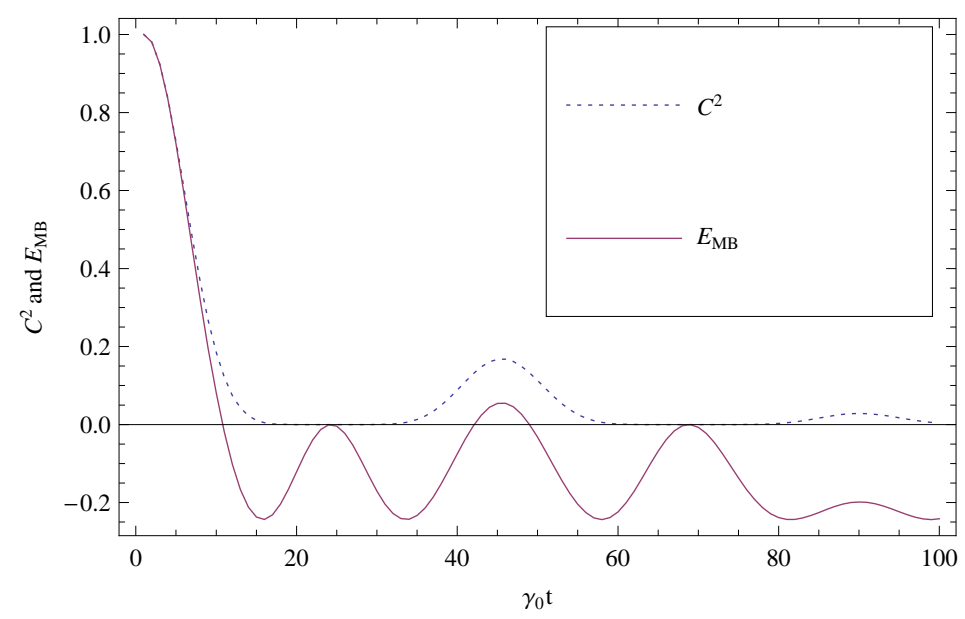

FIG. 7: The concurrence squared and the MB lower bound $E_{M B}$, as a function of $\gamma_{0} t$, for the initial state $\frac{1}{\sqrt{2}}(|00\rangle+|11\rangle)$. All depicted quantities are dimensionless.

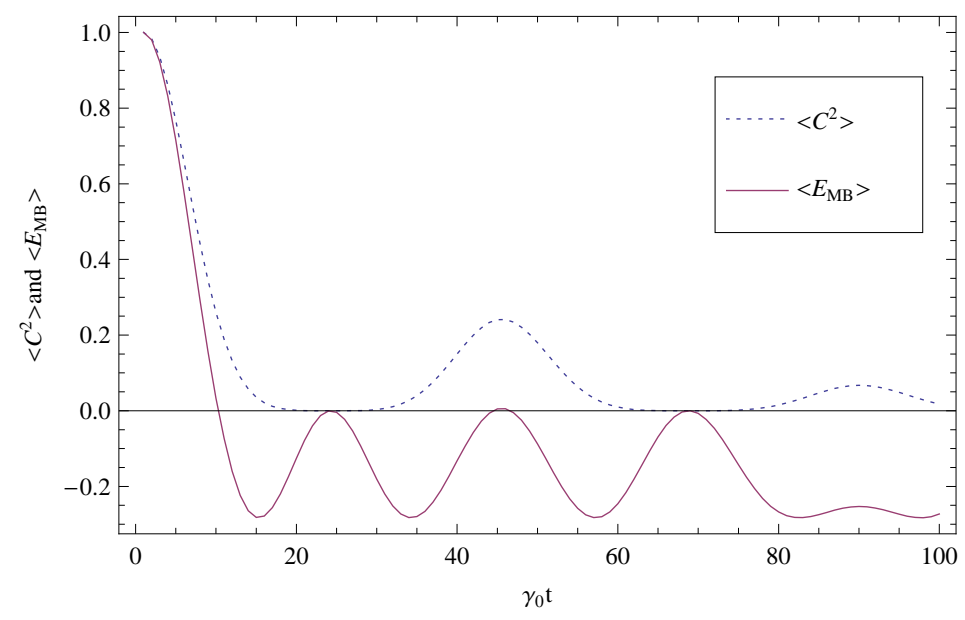

FIG. 8: The averages of the concurrence squared and of the MB lower bound corresponding to initial maximally entangled states, as a function of $\gamma_{0} t$. All depicted quantities are dimensionless. 


\section{B. Entanglement Indicator Based Upon a Local Uncertainty Relationship.}

An interesting connection between quantum separability in bi-partite systems and local uncertainty relations has been pointed out by Hofmann and Takeuchi in [27]. These authors showed that separable states of bi-partite quantum systems comply with certain local uncertainty relations. In particular, all separable states (pure or mixed) of two-qubit systems satisfy

$$
U=\delta\left[\sigma_{1}(A)+\sigma_{1}(B)\right]^{2}+\delta\left[\sigma_{2}(A)+\sigma_{2}(B)\right]^{2}+\delta\left[\sigma_{3}(A)+\sigma_{3}(B)\right]^{2} \geq 4,
$$

where $\sigma_{i}(A), \sigma_{i}(B) i=1,2,3$, are the Pauli matrices corresponding to subsystems $A$ and $B$, respectively, and $\delta O^{2}=\left\langle O^{2}\right\rangle-\langle O\rangle^{2}$ is the uncertainty of the observable $O$. On the basis of (23) we can regard the quantity

$$
\frac{4-U}{4}
$$

as an entanglement indicator. Any state with $(4-U) / 4>0$ is necessarily entangled. On the other hand, if the above quantity is negative, the state may be entangled or separable. The entanglement indicator (24) is of interest because it is based on quantities that are in principle measurable.

There are some particular initial, maximally entangled states for whom the entanglement of the time-dependent state $\rho$ is detected (at least part of the time) by the violation of the uncertainty relation (23). Therefore, for these states the quantity $(4-U) / 4$ exhibits positive values when the state $\rho$ has a large enough amount of entanglement. This behavior can be seen in Fig. 9 for the initial state $\frac{1}{\sqrt{2}}(|01\rangle-|10\rangle)$. The situation is different when one considers the average behavior of the uncertainty sum $U$ over the family of maximally entangled initial states. One can see in Fig. 10 that, on average, the time dependent states arising from maximally entangled initial states do not violate the uncertainty relation (23). It is interesting that, even though these states do exhibit on average (at certain times) a considerable amount of entanglement, they behave strictly as separable states as far as the local uncertainty relation (23) is concerned. 


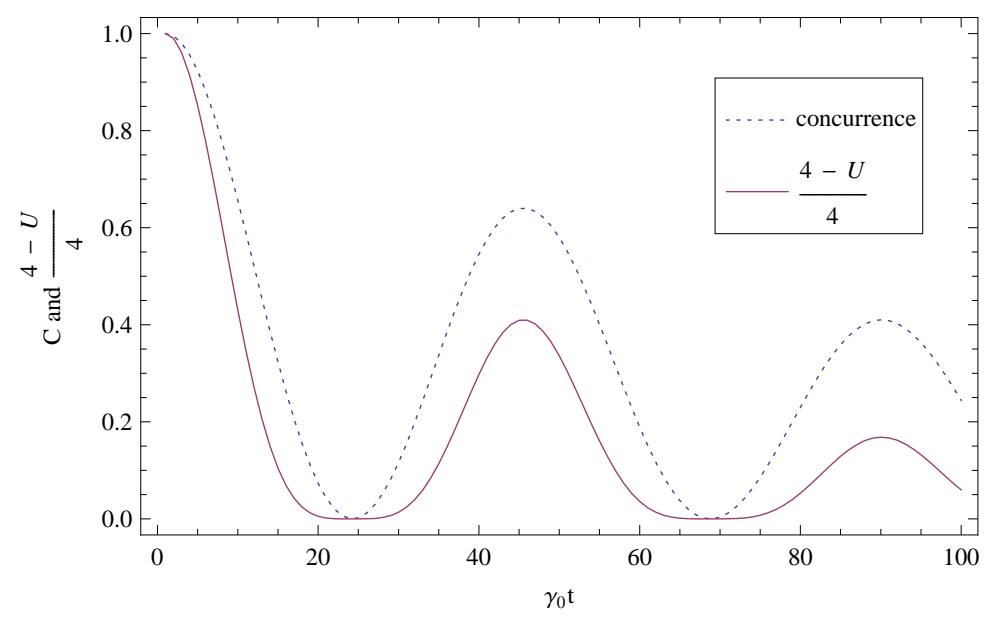

FIG. 9: The uncertainty-based entanglement indicator and the concurrence $C$ against $\gamma_{0} t$, for the initial state $\frac{1}{\sqrt{2}}(|01\rangle-|10\rangle)$. The indicator is set equal to zero if the quantity (24) has a negative value. All depicted quantities are dimensionless.

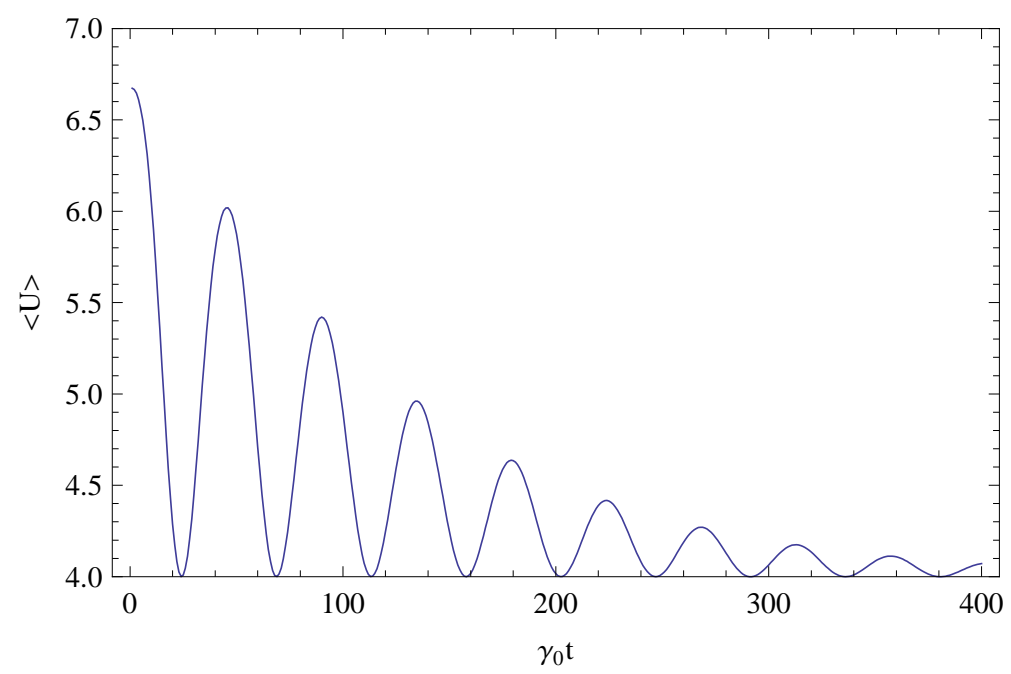

FIG. 10: The average of the uncertainty sum $U$ for initial maximally entangled states as a function of $\gamma_{0} t$. All depicted quantities are dimensionless.

The general trend observed in connection with the entanglement estimators considered here is that they tend to be less successful in detecting entanglement during the entanglement revivals than during the initial time interval of entanglement decrease. This seems to be closely related to the fact that during the entanglement revivals the system under consid- 
eration tends to be more mixed than during the initial entanglement decay. The various manifestations of entanglement tend to be weaker for states of increasing degree of mixedness. This is clearly observed, for instance, in the case of the Minternt-Buchleitner lower bound $E_{M B}$ for the squared concurrence. Indeed, the ability of this quantity to detect entanglement deteriorates when one considers states of increasing mixedness [23].

\section{SOME FEATURES OF THE ENTANGLEMENT DYNAMICS OF SYSTEMS OF THREE AND FOUR QUBITS INTERACTING WITH AN ENVIRONMENT}

In this section we are going to consider the entanglement dynamics of three-qubits systems interacting with an environment in the non-Markovian regime. As in the two-qubits case, we assume that each qubit interacts with its own, independent environment.

In the case of three qubits or more, the GHZ (Greenberger-Horne-Zeilinger) state and the W state constitute two important paradigmatic examples of entangled states. The general expression of the GHZ state is

$$
|G H Z\rangle=\frac{|0\rangle^{\otimes n}+|1\rangle^{\otimes n}}{\sqrt{2}}
$$

where $n$ is the number of qubits. The $n$-qubits $\mathrm{W}$ state is

$$
|W\rangle=\frac{1}{\sqrt{n}}(|100 \ldots 0\rangle+|010 \ldots 0\rangle+\ldots+|000 \ldots 1\rangle) .
$$

Multipartite entanglement measures and their applications have been the focus of considerable research activity in recent years (see [24-26] and references therein). A useful and practical measure for the global amount of entanglement associated with an $n$-qubit state is given by the average of the (bi-partite) entanglement measures corresponding to the $2^{n-1}-1$ possible bi-partitions of the $n$-qubits system [24]. When dealing with mixed states the "negativity" provides an appropriate measure of the amount of entanglement exhibited by a given bi-partition. The negativity is defined as

$$
\text { Neg. }=\frac{1}{2} \sum_{i}\left(\left|\alpha_{i}\right|-\alpha_{i}\right),
$$

where $\alpha_{i}$ are the eigenvalues of the partial transpose matrix associated with a given bipartition. The global, multipartite entanglement measure given by the average (over all 

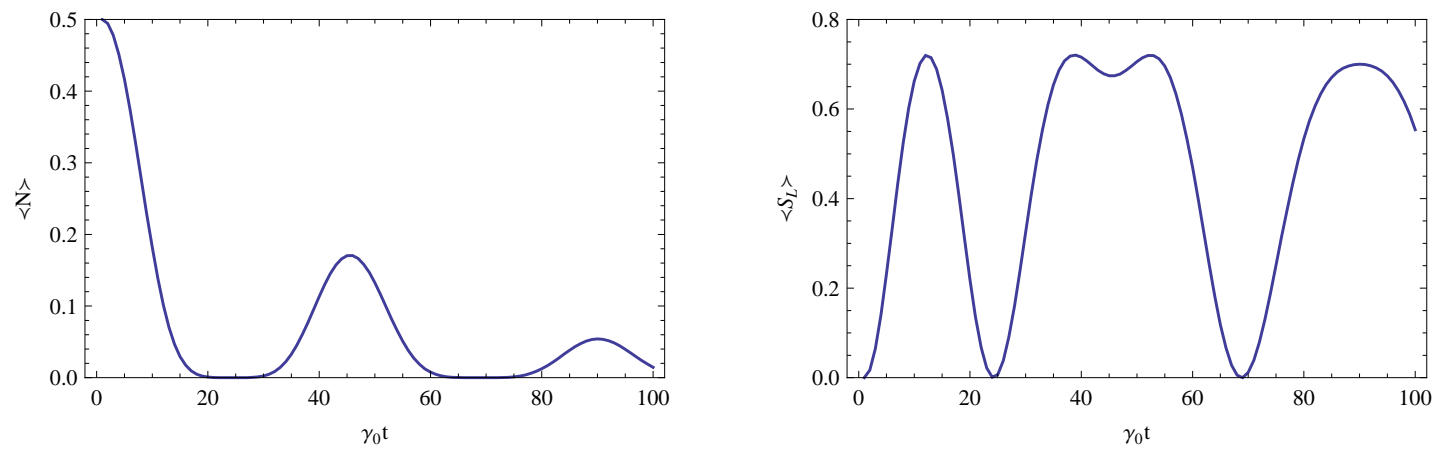

FIG. 11: The average value of the negativity based entanglement measure $N$ (left) and the average value of the linear entropy (right), against the quantity $\gamma_{0} t$, for three-qubit initial states locally equivalent to the GHZ state. All depicted quantities are dimensionless.
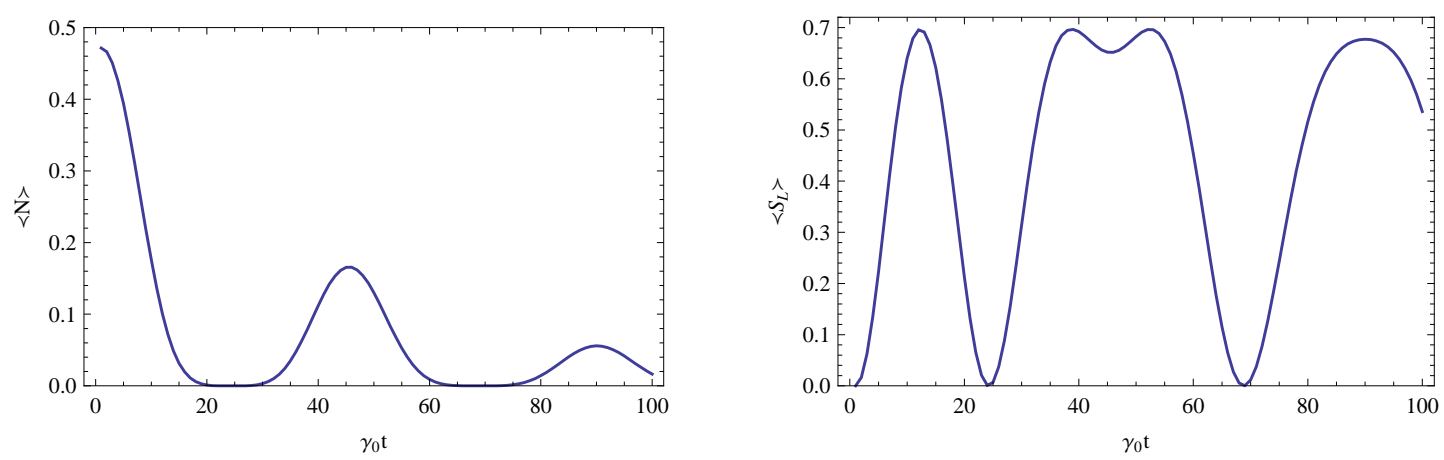

FIG. 12: The average value of the negativity based entanglement measure $N$ (left) and the average value of the linear entropy (right), against the quantity $\gamma_{0} t$, for three-qubit initial states locally equivalent to the $\mathrm{W}$ state. All depicted quantities are dimensionless.

bi-partitions) of the negativity will be denoted $N$.

In this section we consider the average behavior of three and four qubit evolutions with initial states equivalent under local unitary transformations to the $|G H Z\rangle$ or the $|W\rangle$ states. To determine the average behavior associated with initial states locally equivalent to the $n$-qubits $|G H Z\rangle$ state we generate random initial states of the form 


$$
\left(U_{1} \otimes \cdots \otimes U_{n}\right)|G H Z\rangle,
$$

resulting from the action of independent single-qubit unitary operators acting upon each of the $n$ qubits of a multi-qubit system in the $|G H Z\rangle$ state. The single-qubit unitary operators $U_{i}$ (acting on the $i$ th single qubit) are generated randomly, independently and uniformly distributed according to the Haar measure, as described in Section III.A. Then, the average, time dependent properties corresponding to the abovementioned random initial states are computed. A similar procedure was followed to study the average properties of evolutions corresponding to initial states locally equivalent to the $n$-qubits $|W\rangle$ state.

As in the two qubits case, the typical behavior of appropriate families of initial states was studied for three-qubits and and four-qubits systems. The most noticeable difference between the results obtained for two qubits and those obtained for three or four qubits involves the finite time intervals of zero entanglement between entanglement revivals. For initial maximally entangled two-qubits states the finite time intervals of zero entanglement between entanglement revivals disappear when one computes the concomitant average behavior, as shown in Fig. 1. On the contrary, in the case of initial three-qubits states locally equivalent to the $|G H Z\rangle$ or the $|W\rangle$ states the aforementioned intervals of zero entanglement survive after the averaging procedure, as can be appreciated in Figures 11 and 12. This means that the abovementioned finite time intervals of entanglement disappearance are robust features of the entanglement dynamics of three-qubits systems. This is consistent with the fact that the entanglement associated with $n$-qubits systems tends to become more fragile as the number of qubits increases.

Four-qubit systems were also considered. On average, initial states of four-qubits equivalent under local unitary transformations to the $|G H Z\rangle$ and the $|W\rangle$ states behave in a similar way as the corresponding states in the three-qubits case. This can be seen in Figures 13 and 14, where the evolution of the negativity based entanglement measure $N$ is depicted for the four qubit initial states $|G H Z\rangle$ and $|W\rangle$, respectively, together with its average value $\langle N\rangle$ for the families of initial states equivalent under local unitary transformations to those two states. It transpires from Figures 13 and 14 that the time intervals of "dead" entanglement before the entanglement revivals exhibited by the $|G H Z\rangle$ and $|W\rangle$ states are a robust feature of the entire families of initial states locally equivalent to those two states, that is clearly present in their average behaviour. This, again, illustrates the increasing 
entanglement fragility that accompanies an increasing number of qubits.

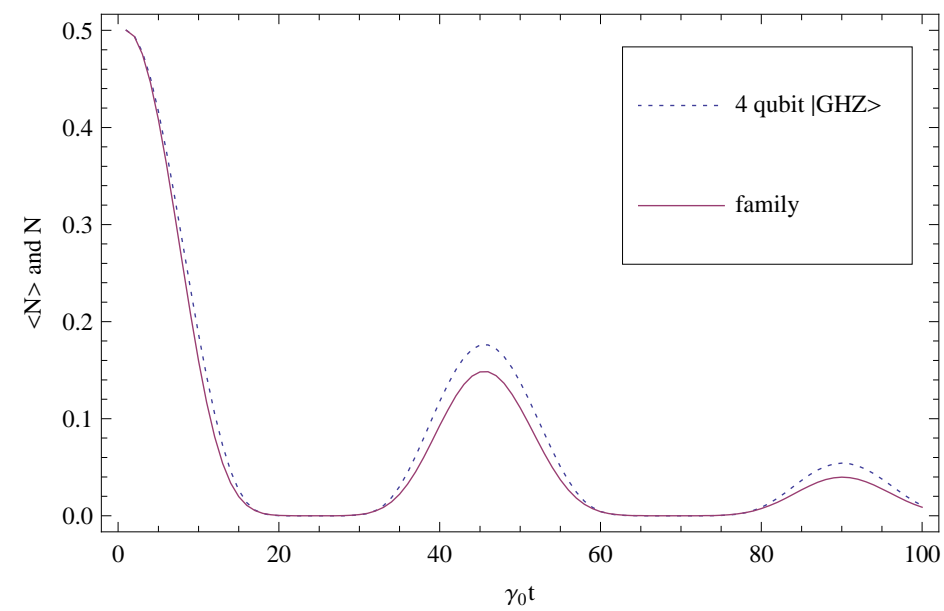

FIG. 13: The negativity based entanglement measure $N$ for the four-qubit initial state $|G H Z\rangle$ (dotted line) and the average $\langle N\rangle$ for the family of initial states equivalent to $|G H Z\rangle$ under local unitary transformations (continuous line) as a function of $\gamma_{0} t$. All depicted quantities are dimensionless.

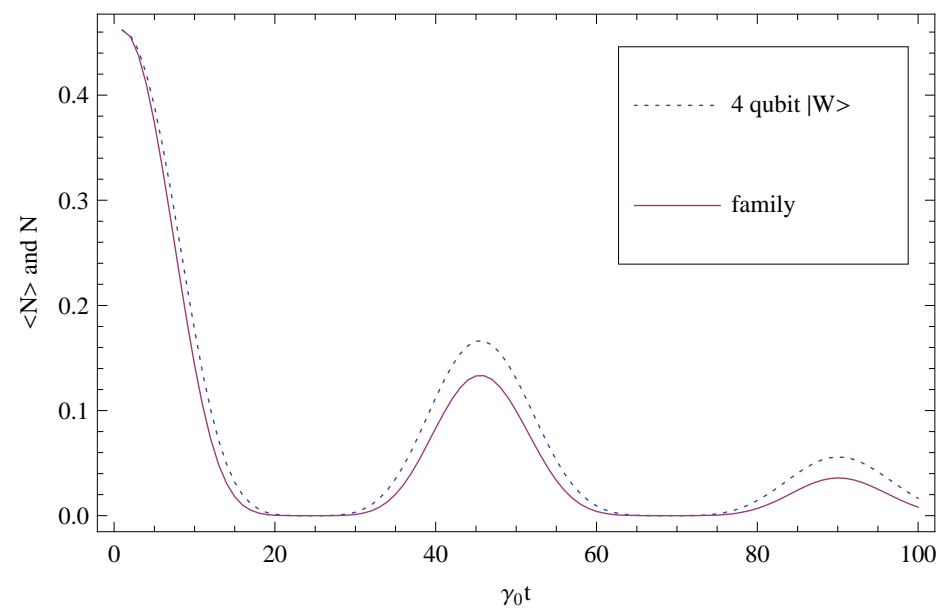

FIG. 14: The negativity based entanglement measure $N$ for the four-qubit initial state $|W\rangle$ (dotted line) and the average $\langle N\rangle$ for the family of initial states equivalent to $|W\rangle$ under local unitary transformations (continuous line) as a function of $\gamma_{0} t$. All depicted quantities are dimensionless. 


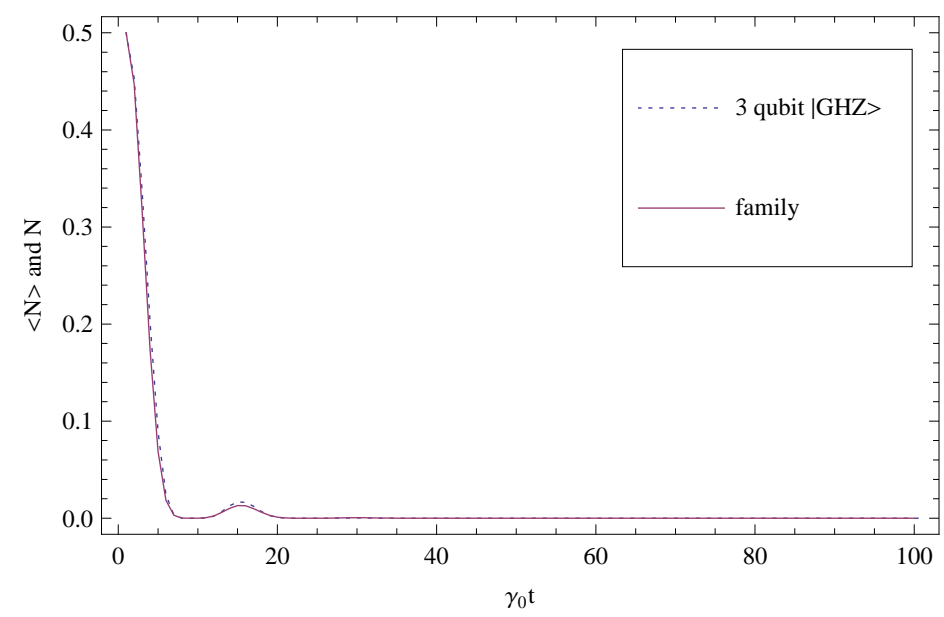

FIG. 15: Evolution of the negativity based entanglement measure $N$ for the three-qubit initial state $\alpha|000\rangle+\sqrt{1-\alpha^{2}}|111\rangle$ with $\alpha^{2}=\frac{1}{3}$ (dotted line) and the average $\langle N\rangle$ for the family of initial states equivalent to the alluded state under local unitary transformations (continuous line), for $\lambda=0.1 \gamma_{0}$. All depicted quantities are dimensionless.

The main purpose of the present effort was to explore some aspects of the entanglement sudden death and subsequent entanglement revival exhibited by multi-qubit systems interacting with an environment in a non-Markovian regime. Consequently, we have focused on the case of $\lambda=0.01 \gamma_{0}$, where the aforementioned phenomena are clearly visible. As a general trend, when one considers larger values of the ratio $\lambda / \gamma_{0}$ (corresponding to less non-Markovian regimes) the alluded phenomena are less pronounced, with shorter periods of "dead" entanglement. However, we have also considered the case $\lambda=0.1 \gamma_{0}$ and our numerical results indicate that the robustness of the periods of dead entanglement before entanglement revivals for systems of more than two qubits also holds for smaller values of the ratio $\lambda / \gamma_{0}$, even if the lengths of these periods are much shorter than in the $\lambda=0.01 \gamma_{0}$ case.

For example, the behaviour of the negativity based entanglement measure $N$ for the three qubit initial state (with $\alpha^{2}=\frac{1}{3}$ )

$$
\alpha|000\rangle+\sqrt{1-\alpha^{2}}|111\rangle
$$

together with its average value $\langle N\rangle$ for the family of initial states equivalent under local unitary transformations to (29), are plotted in Figure 15. It is clear from this Figure that the 
period of zero entanglement between the entanglement sudden death and its sudden revival exhibited by the initial state (29) is also present on the average behavior corresponding to the initial states locally equivalent to (29).

\section{CONCLUSIONS}

We have explored some entanglement-related features of the dynamics of two-qubits, three-qubits, and four-qubits systems interacting with a non-Markovian environment. Our main goal was to explore some entanglement properties of the alluded systems related to the phenomena of entanglement sudden death followed by entanglement sudden revival, which constitute remarkable effects appearing in the alluded systems for small enough values of the ratio $\lambda / \gamma_{0}$. For these reasons, we have focused on a non-Markovian case characterized by $\lambda=0.01 \gamma_{0}$, and our conclusions correspond mainly to this kind of scenarios which are well into the non-Markovian regime.

We have focused upon the average, typical behavior associated with some relevant families of initial states, such as the set of maximally entangled two-qubit states, or the states locally equivalent to the $|G H Z\rangle$ state in the three-qubits and four-qubits cases.

In the case of two qubits the average, typical behavior corresponding to maximally entangled initial states, or the one corresponding to pure, partially entangled states with a given amount of entanglement, doesn't have finite time intervals of zero entanglement between entanglement revivals as is the case for some particular initial states belonging to the alluded families. On the contrary, when investigating the dynamics of entanglement associated with the families of initial states of three or four qubits locally equivalent to the $|G H Z\rangle$ and to the $|W\rangle$ states, we found that the finite intervals of zero entanglement are still present in the average behavior. Consequently, the phenomena of entanglement sudden death and subsequent entanglement revival are robust properties of the evolutions associated with the abovementioned families of initial states of three or four qubits. These features of the entanglement dynamics of three qubits and four qubits systems are consistent with the fact that, in general, entanglement becomes more fragile as the number of qubits of a system increases.

We investigated the connection between the time evolution of the amount of entanglement exhibited by the multi-qubit system on the one hand, and its global degree of mixedness 
(as measured by the total linear entropy $S_{L}$ ) on the other one. As a general trend, the entanglement exhibited by multi-partite quantum systems tends to decrease as the degree of mixedness increases. However, except for the initial period of entanglement decrease, the systems considered here tend to exhibit the largest amount of entanglement simultaneously with the largest degrees of mixedness. Indeed, during the entanglement revivals entanglement and mixedness tend to increase and decrease together. We have determined the trajectory followed by the multi-qubits systems (for various families of initial states) in the $\left(\left\langle S_{L}\right\rangle-\langle C\rangle\right)$-plane. In all the cases studied, for two qubits, three qubits and four qubits, these trajectories exhibit the shape of an inverted "C" with two branches, one corresponding to the initial phase of entanglement decrease, and the second branch corresponding to the entanglement revivals. In the case of maximally entangled initial states of two-qubits, the first branch is relatively close to the MEMS curve, while the second branch departs drastically from it.

In the case of two qubits the behavior of two entanglement indicators based upon measurable quantities was also examined. We considered the Minternt-Buchleitner lower bound $E_{M B}$ for the squared concurrence and an entanglement indicator based on the violation of a local uncertainty relation. For the initial state $\frac{1}{\sqrt{2}}(|00\rangle+|11\rangle)$, the quantity $E_{M B}$ exhibited "sudden death" and one "revival". On the other hand, the average behavior of $E_{M B}$ corresponding to maximally entangled initial states has sudden death, but no revival. During the the period of entanglement decrease $E_{M B}$ provides a reasonable estimate for the squared concurrence. The estimator based on the violation of local uncertainty relations does detect the entanglement of the evolving two-qubit state for some initial conditions. However, its average behavior for initial maximally entangled states corresponds to separable states. These findings are consistent with the results reported by Bellomo et al. in [12], where it was shown that even at times when the two-qubits system still has a considerable amount of entanglement it behaves "classically", as far as the Bell inequalities are concerned. Our present results show that the time dependent state of the two-qubits system, particularly during the entanglement revivals, also fails to exhibit other manifestations of entanglement, such as positive values of the Minternt and Buchleitner indicator $E_{M B}$, or the violation of local uncertainty relations. 


\section{Acknowledgments}

The financial assistance of the National Research Foundation (NRF; South African Agency) towards this research is hereby acknowledged. Opinions expressed and conclusions arrived at, are those of the authors and are not necessarily to be attributed to NRF. This work was partially supported by the Projects FQM-2445 and FQM-207 of the Junta de Andalucia (Spain).

[1] Bengtsson I and Zyczkowski K, 2006 Geometry of Quantum States: An Introduction to Quantum Entanglement (Cambridge, Cambridge University Press)

[2] Jaeger G, 2007 Quantum Information (New York, Springer)

[3] Nielsen M A and Chuang I L, 2000 Quantum Computation and Quantum Information (Cambridge University Press, Cambridge)

[4] Schlosshauer M, 2005 Rev. Mod. Phys. 761267

[5] Gemmer J, Michel M, and Mahler G, 2004 Quantum Thermodynamics (Springer-Verlag, Berlin)

[6] Maccone L, 2009 Phys. Rev. Lett. 103080401

[7] Giovannetti V, Lloyd S, and Maccone L, 2006 Phys. Rev. Lett. 96010401

[8] Yu T and Eberly J H, 2004 Phys. Rev. Lett. 93140404

[9] Al-Qasimi A and James D F V, 2008 Phys. Rev. A 77012117

[10] Bellomo B, Lo Franco R, and Compagno G, 2007 Phys. Rev. Lett. 99160502

[11] Bellomo B, Lo Franco R, and Compagno G, 2008 Phys. Rev. A 77032342

[12] Bellomo B, Lo Franco R, and Compagno G, 2008 Phys. Rev. A 78062309

[13] Cui W, Xi Z and Pan Y, 2009 J. Phys. A: Math. Theor. 42025303

[14] Cui W, Xi Z and Pan Y, 2009 J. Phys. A: Math. Theor. 42155303

[15] Hamadou-Ibrahim A, Plastino A R, and Plastino A, 2009 Braz. J. Phys. 39408

[16] Almeida M P, de Melo F, Hor-Meyll M, Salles A, Walborn S P, Souto Ribeiro P H, Davidovich L, 2007 Science 316579

[17] Borras A, Majtey A P, Plastino A R, Casas M, and Plastino A, 2009 Phys. Rev. A 79022108

[18] Breuer H P and Petruccione F, 2002 The Theory of Open Quantum Systems (Oxford University 
Press, Oxford)

[19] Garraway M, 1997 Phys. Rev. A 552290

[20] Zyczkowski K, Horodecki P, Horodecki M, and Horodecki R, 2001 Phys. Rev. A 65012101

[21] Batle J, Casas M, Plastino A R, Plastino A, 2002 Phys. Lett. A 296251

[22] Mintert F and Buchleitner A, 2007 Phys. Rev. Lett. 98140505

[23] Borras A, Majtey A P, Plastino A R, Casas M and Plastino A, 2009 Phys. Rev. A 79022112

[24] Brown I D K, Stepney S, Sudbery A and Braunstein S L, 2005 J. Phys. A: Math. Gen. 38 1119

[25] Borras A, Plastino A R, Batle J, Zander C, Casas M and Plastino A, 2007 J. Phys. A: Math. Theor. 4013407

[26] Zander C, Plastino A R, Plastino A, and Casas M, 2007 J. Phys. A: Math. Theor. 402861

[27] Hofmann H F and Takeuchi S, 2003 Phys. Rev. A 68, 032103

[28] Munro W J, James D F V, White A G, and Kwiat P G, 2001 Phys. Rev. A 64030302

[29] Naudts J and Verhulst T, 2007 Phys. Rev. A 75, 062104

[30] Borras A, Plastino A R, Casas M, and Plastino A, 2008 Phys. Rev. A 78052104 